\title{
Clinical consensus of emergency airway management
}

\author{
Feng Sun, Ya Wang, Shicheng Ma, Huadong Zhu, Xuezhong Yu, Jun Xu; on behalf of Chinese \\ Collaboration Group for Emergency Airway Management*
}

Emergency Department, Peking Union Medical College Hospital, Beijing 100730, China

Correspondence to: Jun Xu. Emergency Department, Peking Union Medical College Hospital, No. 1 Shuangfuyuan Hutong, Dongcheng District, Beijing 100730, China. Email: xujunfree@126.com.

\begin{abstract}
Airway management is a common and key method to maintain and improve external respiration function of patients. Emergency physicians need a more appropriate guide to airway management. We concisely concluded current circumstances of Chinese emergency airway management. Then, we raised four principles: (I) priority to ventilation and oxygenation; (II) evaluation before intubation; (III) higher level of preparation (de-escalation); (IV) simplest (and least potentially harmful) form of intubation. We raised "CHANNEL" flow to direct initial emergency airway management and an algorithm was showed for emergency physicians understanding key points of airway management and further making medical decision. Finally, we introduced pharmacology of airway management.
\end{abstract}

Keywords: Emergency medicine; airway management; intubation; tracheostomy

Submitted Aug 31, 2017. Accepted for publication Oct 13, 2017.

doi: $10.21037 /$ jtd.2017.10.79

View this article at: http://dx.doi.org/10.21037/jtd.2017.10.79

\footnotetext{
* List of Chinese Collaboration Group for Emergency Airway Management: Xuezhong Yu (Peking Union Medical College Hospital, Beijing, China); Ying Deng (The Second Affiliated Hospital of Harbin Medical University, Harbin, China); Zhong Wang (Beijing Tsinghua Changgung Hospital, Beijing, China); Guoxing Wang (Beijing Friendship Hospital, Capital Medical University, Beijing, China); Zhongqiu Lu (The First Affiliated Hospital of Wenzhou Medical University, Wenzhou, China); Yingping Tian (The Second Hospital of Hebei Medical University, Shijiazhuang, China); Huadong Zhu (Peking Union Medical College Hospital, Beijing, China); Jihong Zhu (Peking University People's Hospital, Beijing, China); Yong Li (Beijing Anzhen Hospital, Beijing, China); Lishan Yang (General Hospital of Ningxia Medical University Yinchuan, China); Jinsong Zhang (Jiangsu Province People's Hospital, Nanjing, China); Hong Zhang (The First Affiliated Hospital of Anhui Medical University, Hefei, China); Mao Zhang (The Second Affiliated Hospital of Zhejiang University, Hangzhou, China); Xinchao Zhang (Beijing Hospital, Beijing, China); Yuguo Chen (Qilu Hospital of Shandong University, Jinan, China); Libo Chen (Wuhan Union Hospital, Wuhan, China); Qiming Feng (The 6th People's Hospital of Shanghai, Shanghai, China); Liangliang Zheng (Beijing Hospital, Beijing, China); Xiaodong Zhao (The First Affiliated Hospital of PLA General Hospital, Beijing, China); Yimin Zhu (Hunan Province People's Hospital, Changsha, China); Yanfen Chai (Tianjin Medical University General Hospital); Ming Gu (Peking Union Medical College Hospital, Beijing, China); Chuanyun Qian (The First Affiliated Hospital of Kunming Medical University, Kunming, China); Suyun Qian (Beijing Children's Hospital, Capital Medical University, Beijing, China); Wei Guo (Beijing Tiantan Hospital, Capital Medical University, Beijing, China); Yu Cao (West China Hospital, Chengdu, China); Jun Xu (Peking Uniona Medical College Hospital, Beijing, China); Shengyong Xu (Peking Union Medical College Hospital, Beijing, China); Xianquan Liang (The Second People's Hospital of Guiyang, Guiyang, China); Lu Liang (Affiliated Hospital of Hebei University, Baoding, China); Peng Peng (The First Affiliated Hospital of Xinjiang Medical University, Urumqi, China); Yingjie Chu (Henan Province People's Hospital , Zhengzhou, China); Hong Zhan (The First Affiliated Hospital of Sun Yat-sen University, Guangzhou, China); Hui Xiong (Peking University First Hospital, Beijing, China); Tanshi Li (The General Hospital of the People's Liberation Army, Beijing, China); Shuming Pan (Xin Hua Hospital Affiliated to Shanghai Jiao Tong University School of Medicine, Shanghai, China); Jiayuan Dai (Peking Union Medical College Hospital, Beijing, China); Feng Sun (Peking Union Medical College Hospital, Beijing, China); Ya Wang (Peking Union Medical College Hospital, Beijing, China); Shicheng Ma (Peking Union Medical College Hospital, Beijing, China).
} 


\section{Background}

Emergency airway management is an essential skill for emergency physicians. The guidelines for airway management written by anesthesiologists (1) are not wholly applicable or appropriate in emergency clinical conditions. Based on the unique features of emergency airway management, we put forward this clinical consensus policy based on four emergency medicine principles: (I) priority to ventilation and oxygenation, (II) evaluation before intubation, (III) higher level of preparation (de-escalation), (IV) simplest (and least potentially harmful) form of intubation. This policy aims to standardize the management of the emergency airway.

\section{Characteristics of the emergency airway}

Urgency and unpredictability are primary characteristics of the emergency airway. The following factors further increase the difficulty of emergency airway management: (I) lack of information: unlike anesthesiologists in the operation room, emergency physicians often cannot get information such as detailed medical history or have time to complete a thorough physical examination before needing to act. A clear process should be followed so that the airway can managed rapidly; (II) lack of preparation: the decision to establish an artificial airway is often made suddenly and preparation time is usually short, which means a comprehensive preparation cannot be relied upon at every intubation; (III) lack of compliance: emergency patients may have difficulty in cooperating. The patient may have recently eaten but still may need an artificial airway immediately, and will therefore more easily vomit or aspirate during the intubation. Sometimes there's only one chance for physicians to establish the artificial airway; (IV) lack of equipment: equipped with simple and basic instruments, Chinese emergency doctors often have few options when they encounter a difficult airway; (V) lack of standardization: individual experiences and generally accepted processes in every emergency room frequently vary, and some emergency physicians have little, even no training in dealing with a difficult airway. Therefore, it is necessary to develop a standardized airway management protocol.

\section{Concepts}

\section{Difficult emergency airway}

This is defined as the clinical situation in which a conventionally trained emergency doctor encounters difficulty in bag-valve-mask (BVM), intubation, or both.

\section{Difficult intubation}

\section{Difficult laryngoscopy}

Unable to see any portion of the vocal cords after several attempts by conventional laryngoscopy (Laryngeal view grade III-IV).

\section{Difficult intubation}

Multiple attempts are required for intubation, with or without tracheal pathology.

\section{Urgent airway}

As long as there's difficulty in BVM, with or without intubation, it can be defined as an Urgent airway. Patients are more likely to be in a hypoxic state (1).

\section{Clinical decision-making strategies for emergency airway management}

Step 1: ensure ventilation and oxygenation, and assess airway conditions on the basis of the "CHANNEL" principle as described below. Keep in mind: the safety of the patient is always the primary goal.

Step 2: ascertain airway conditions and establish an artificial airway. At this stage, check the airway with laryngoscopy, and decide which level of practitioners should finish the intubation and which equipment should be used following the principle of 'De-escalation'. An airway management cart is recommended to ensure every piece of equipment is at hand when needed. If conditions permit, intubating following the procedure of Rapid Sequence Intubation is recommended. While dealing with a difficult airway, follow the principle of 'priority to ventilation and oxygenation' and avoid excessive blind attempts. The whole decision-making of airway management follows the principle of using the 'simplest and least injurious method of intubation' and video laryngoscopes are recommended.

A detailed flow chart of emergency airway management is shown in Figure 1.

\section{“CHANNEL" principle}

Crash airway

Crash airway refers to patients in cardiopulmonary arrest, 


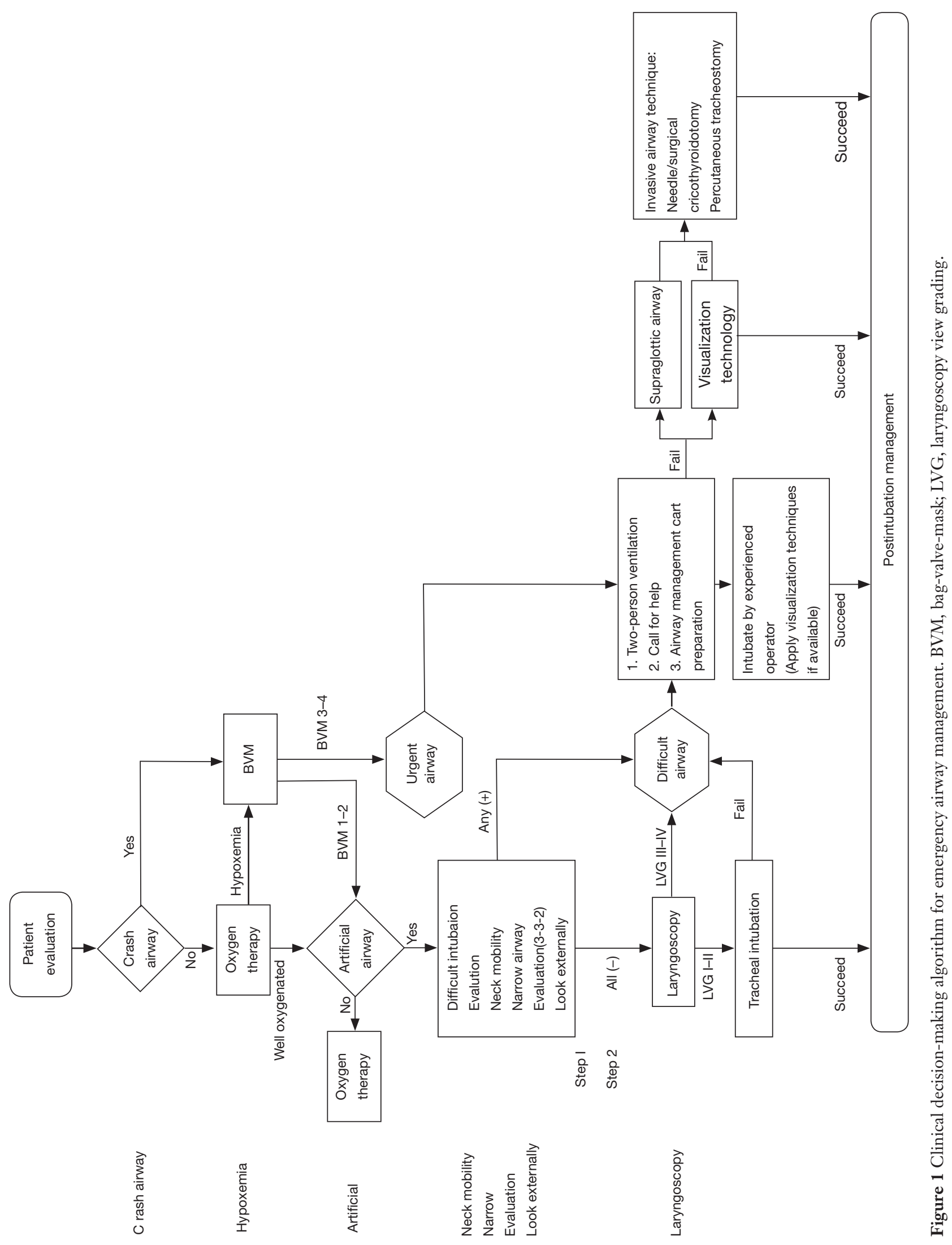


deep coma or near death, who can't maintain ventilation and oxygenation. A crash airway should be managed quickly as per the flow-chart above (i.e., BVM ventilation and rapidly moving to laryngoscopy).

\section{Hypoxemia}

A primary goal of emergency airway management is to correct hypoxemia. In patients with stable spontaneous respiration, nasal cannulas and high-flow oxygen devices (such as non-rebreather masks or venturi mask) are common means of delivering oxygen therapy. In patients with unstable spontaneous respiration as well as hypoxemia despite the above treatment, BVM ventilation is demanded. All hypoxemia patients need proper ventilation to avoid carbon dioxide retention.

Persistent hypoxemia despite using the above oxygen therapies should be managed as an urgent airway. In these cases, an artificial airway should be built immediately according to the urgent airway process, and invasive airway devices should be ready.

BVM ventilation: mask seal and an open airway are the two key points of BVM ventilation. Situations suggestive of difficult BVM include: the elderly ( $>55$ years old), obesity (body mass index $>26 \mathrm{~kg} / \mathrm{m}^{2}$ ), edentulousness (no teeth), presence of beards, and a history of sleep apnea (2). The difficulty grade (grades 1-4) of BVM is described as Grade 1: good ventilation with bag-mask in the supine position; Grade 2: good ventilation a through oral/nasopharyngeal airway with onehanded ventilation; Or with both hands holding the mandible to improve seal; Grade 3: unable to achieve good ventilation with the above-mentioned methods. Two-person ventilation is required to maintain $\mathrm{SpO}_{2} \geq 90 \%$; Grade 4: impossible to maintain $\mathrm{SpO}_{2} \geq 90 \%$ even with two-person ventilation. Good ventilation is defined as appropriate resistance during positive pressure ventilation with BVM (airway resistance $\leq 20 \mathrm{cmH}_{2} \mathrm{O}$, at least 3 times), thoracoabdominal fluctuation is normal, and the wave of end-tidal carbon dioxide $\left(\mathrm{ETCO}_{2}\right)$ partial pressure appears regularly, excluding factors such as poor BVM fit and/or excessive leakage. Two-person ventilation refers to the condition in which two practitioners are involved, use oropharyngeal/nasopharyngeal airway and one operator fastens the BVM while the other ventilates powerfully. Patients rated grades 1 and 2 can get usually be well-ventilated with BVM techniques, while grades 3 and 4 are rather difficult $(1,3)$. If difficult BVM ventilation is encountered in the course of single-person operation, manually opening the airway, using oral- or nasopharyngeal airways, and/or two-person operation can potentially improve ventilation.
Sellick maneuver: for patients losing intact airway selfprotection, especially accompanied by a full stomach, the Sellick maneuver is recommended to prevent aspiration and regurgitation (4). Press cricoid cartilage with index finger and thumb under a force of $20-44$ newtons ( 10 newtons $\approx 1 \mathrm{~kg}$ ) towards the vertebrae to occlude the esophagus. Apply cricoid pressure until endotracheal intubation is completed and cuff is inflated (5-7). If intubation or BVM are adversely affected by this motion, cricoid pressure should be released (8).

\section{Artificial airway}

For patients who are capable of maintaining ventilation and oxygenation, indications for intubation should still be evaluated.

There are both non-invasive and invasive types of artificial airways. Non-invasive airways include: endotracheal intubation, and supraglottic techniques [laryngeal mask airways (LMAs)], etc. Invasive airways include: tracheotomy, needle or surgical cricothyroidotomy, etc. Among the above, endotracheal intubation is the most common method of creating an artificial airway. Indications for endotracheal intubation include: inability to protect the airway or maintain airway patency, failure to adequately achieve ventilation or oxygenation, and anticipation of a deteriorating clinical course that will eventually lead to the above-mentioned situations. There is no absolute contraindication to the above procedures when attempting to rescue patients. Relative contraindications are trachea-laryngeal edema, acute pharyngitis/laryngitis, tracheal submucosal hematoma, tracheal rupture, and severe coagulation dysfunction.

\section{Neck mobility}

Neck mobility is essential for positioning the patient for optimal direct laryngoscopy. Neck stiffness/injury/fixation, or an uncooperative patient refusing to position their neck properly increases the difficulty of endotracheal intubation (9). Visualization techniques like video laryngoscopy are recommended in this condition.

\section{Narrowing}

Any condition resulting in decreased endotracheal diameter, such as extratracheal compression (tumor, local abscess, hematoma, etc.), intratracheal foreign body, tracheal diseases (local radiotherapy, scar healing, etc.) would increase the difficulty of intubation.

\section{Evaluation}

It is required to adjust mouth axis, pharynx axis and larynx 

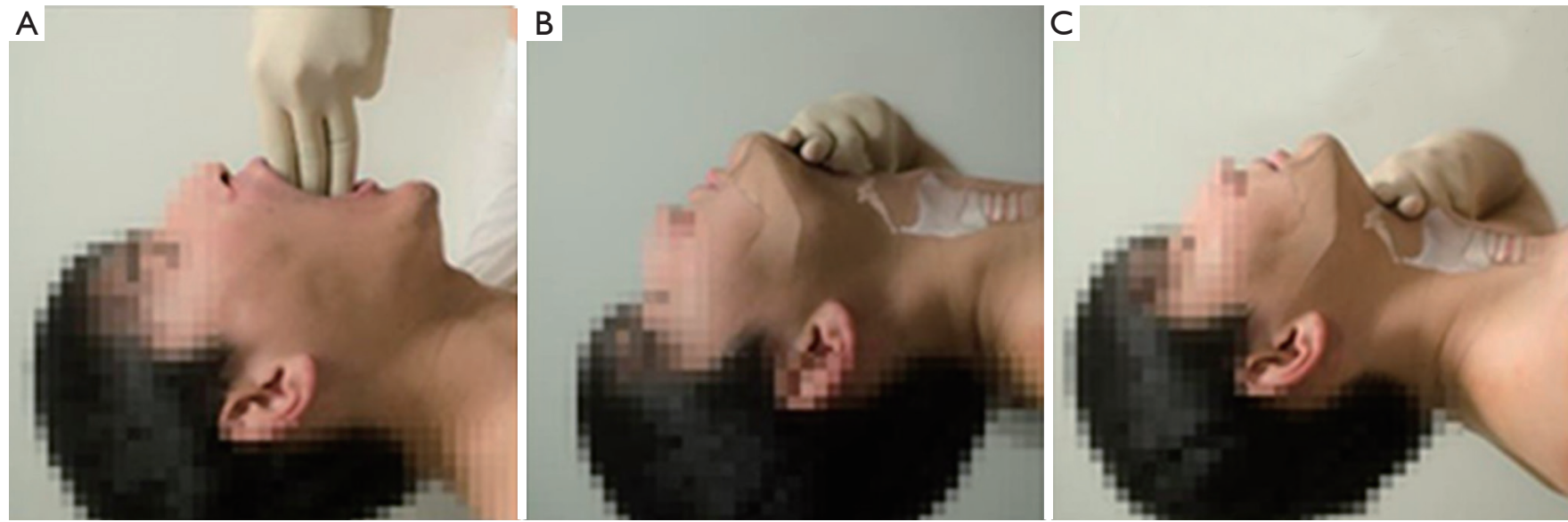

Figure 2 The 3-3-2 Rule. (A) More than 3 fingers between the open incisors, indicating patient's mouth opens adequately to permit the laryngoscope to reach the airway; (B) more than 3 fingers along from mentum to hyoid bone, which indicates enough space for intubation; (C) more than 2 fingers from the laryngeal prominence to hyoid bone, less than 2 fingers indicate high position of pharynx in the neck and likely difficult exposure with direct laryngoscope.

axis to be aligned as much as possible when conducting orotracheal intubation. The 3-3-2 Rule (10) is used to evaluate the correlation of these three axes. Inability to meet the 3-3-2 Rule indicates a likely difficult glottis exposure under direct laryngoscopy (Figure 2).

If permitted, use the modified Mallampati scale to evaluate the structure of the pharynx (Class 1: soft palate, fauces, uvula, and tonsillar pillars visible; Class 2: soft palate, fauces, and uvula visible; Class 3: soft palate, and base of uvula visible; Class 4: Soft palate not visible.). The higher the grade, the more difficult it is to view under direct laryngoscopy. Grades 3 and 4 suggest a difficult airway (11).

\section{Look externally}

Look externally to examine for signs of a potentially difficult intubation, such as a short neck, obesity, a receding mandible, long canine teeth, traumatic deformities, etc.

\section{Laryngoscopy technique}

\section{Laryngoscopy view grading}

After proper analgesia, sedation as well as muscle relaxation, the upper airway can be further evaluated by the laryngoscopic view grading system (Grade I: visualization of the entire laryngeal aperture; Grade II: visualization of only the posterior commissure of the laryngeal aperture; Grade III: visualization of only the epiglottis; Grade IV: visualization of only the soft palate) (Figure 3), based on varying degrees of laryngeal aperture exposure. Grades 1 and 2 suggest easy intubation with direct laryngoscopy, grade 3 is likely difficult, and grade 4 is extremely difficult (12). Both grade 3 and grade 4 indicate a likely difficult airway.

\section{First attempt}

For laryngoscopic views of grades I and II under direct laryngoscopy, practitioners could attempt direct endotracheal intubation. Stop repeated attempts if difficulty is encountered. If the intubation attempt fails, treat the situation as a difficult airway immediately.

\section{Difficult airway management}

If a difficult airway is encountered, start the difficult airway management algorithm: ensure ventilation and oxygenation by BVM, and concurrently seek help from an experienced operator. An airway management cart containing alternative airway approaches should be ready.

\section{Noninvasive airway techniques \\ Visualization technology}

Visualization technology has been widely applied in clinical practice. It decreases intubation difficulty by improving the view of the glottis. Common devices include video laryngoscopes, fiberoptic bronchoscopes, mirrored direct visualization devices, etc.

\section{Supraglottic airway devices}

Supraglottic airway devices are used to keep the upper 

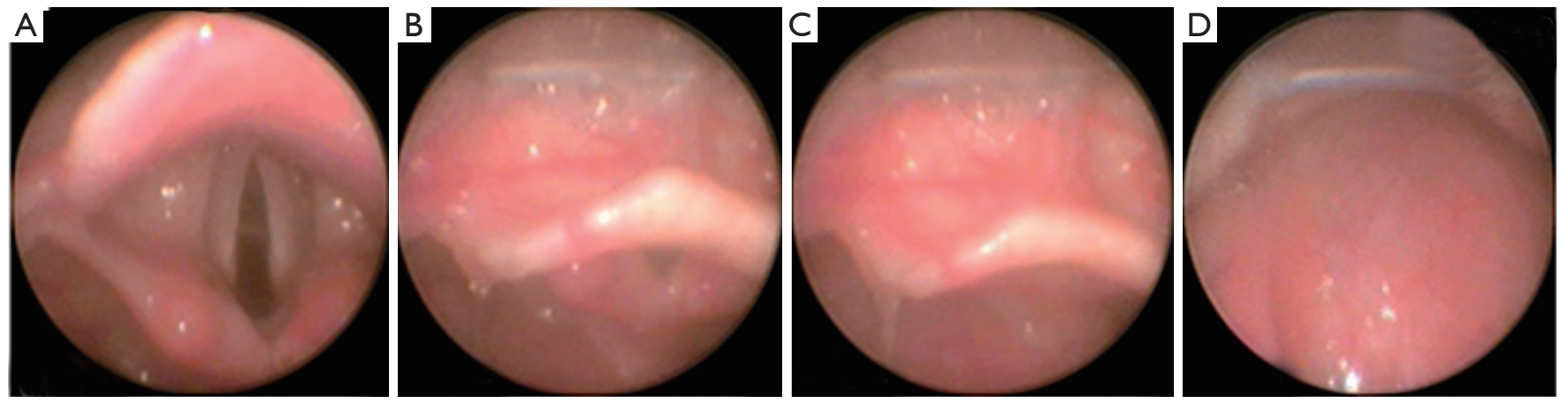

Figure 3 Laryngoscopy view grading system. (A) Visualization of the entire laryngeal aperture (Grade I); (B) visualization of only the posterior commissure of the laryngeal aperture (Grade II); (C) visualization of only the epiglottis (Grade III); (D) visualization of only the soft palate (Grade IV).

airway open to provide unobstructed ventilation, which are employed at critical time when patients cannot be intubated or ventilated by BVM. Contraindications for use of supraglottic airway devices include obstructive airway diseases, traumatized airways, etc. Supraglottic airway devices should be used under sedation to reduce pharyngeal spasms/reflexes that may worsen ventilation.

The LMA is a common supraglottic airway device used as an aid for intubation of the emergent airway (13). The LMA can be used for intubation when a difficult laryngoscopic view or difficult BVM is encountered to maintain adequate ventilation in patients with severe hypoxia, and facilitate subsequent treatment (14). In terms of safety, the LMA does not increase the risk of aspiration (15) but is easier to dislocate compared with endotracheal intubation, therefore, careful fixation should be guaranteed.

\section{Other assisted intubation devices}

Include gum elastic bougies, lighted stylet, intubating LMA, esophagotracheal combitubes, and others.

\section{Invasive airway techniques \\ Needle/percutaneous cricothyroidotomy}

The needle/percutaneous cricothyroidotomy procedure can rapidly establish a definitive but temporary airway. Cricothyroidotomy is indicated when oral or nasal intubation is impossible and/or BVM cannot maintain adequate oxygenation, such as foreign body obstruction, supra-laryngeal trauma, injury (inhalation/thermal/ corrosive) of the upper respiratory tract, angioedema, upper respiratory tract hemorrhage, acute laryngeal obstruction from epiglottis/pseudomembranous laryngitis. It is faster and easier to perform than a tracheotomy in terms of requiring less surgical skill and in its ease of learning (16).

Contraindications include distorted neck anatomy, laryngeal or tracheal rupture, distal trachea communicating with the mediastinum, laryngeal diseases (stenosis, tumor, infection, etc.), children less than 8 years old, coagulation dysfunction (relative), and inexperience (relative).

\section{Tracheotomy}

Equivalent to endotracheal intubation, tracheotomy could establish both a definite and permanent airway when oral or nasal intubation is impossible. In emergency setting, percutaneous tracheostomy is recommended as it is much more rapidly performed.

\section{Pharmacology of airway management}

Laryngoscope insertion and endotracheal intubation can induce strong discomfort and gag reflexes in conscious patients, which result in noncompliance and intolerance, the stimulation of sympathetic nerves and exacerbating underlying pathologies, such as reactive airway diseases or cardiovascular diseases, which may increase the risks of intubation. We recommend administrating the appropriate 'induction' agents to optimize intubating conditions. Agents of rapid onset and short effect are the best choices. Also, it is not recommended to administer one kind of drug class as a replacement for another - analgesia cannot be replaced by sedation or paralysis and vice versa.

\section{Analgesia}

Airway manipulation induces pain and discomfort. Appropriate analgesia is necessary. Most analgesic agents have side effects of respiratory depression. Thus, drugs with rapid onset and short duration, such as remifentanil, alfentanil, 
fentanyl and, possibly, morphine are recommended.

\section{Sedation}

We recommend inducing moderate sedation to improve the compliance, relieve the anxiety and offset the unpleasant feelings of neuromuscular blocking agents. Drugs of rapid onset and short duration, like propofol, etomidate, and midazolam are recommended.

\section{Paralysis}

Neuromuscular blocking agents improve intubation conditions, abolish laryngeal reflexes, relax muscles, increase chest compliance and facilitate BVM, and is strongly recommended in difficult intubations. These agents will stop any respiratory drive, however, so can be particularly dangerous in a patient that is difficult to ventilate. This can quickly devolve into the dreaded 'can't intubate, can't ventilate' scenario. Agents of rapid onset like succinylcholine and rocuronium are recommended, and should be used under sedation.

\section{Post-intubation management}

\section{Confirmation of endotracheal intubation placement}

To ensure the tube is in the trachea, traditional methods, such as bilateral chest auscultation (fourth intercostal space along the midaxillary line) and stomach auscultation, visualization of condensation on the interior of the endotracheal tube, presence of chest wall rise, and others, are not always reliable. Therefore, it is necessary to combine at least two methods for a reliable judgment of intubation success (17). We recommend using $\mathrm{ETCO}_{2}$ detectors as the primary choice to verify the position of the endotracheal tube. In most cases, persistent detected $\mathrm{ETCO}_{2}$ indicates that the tube is in the airway. It should be noted that $\mathrm{ETCO}_{2}$ cannot determine the depth of the endotracheal tube $(18,19)$.

The next step is to ensure the tube is between the vocal chords and the carina. A single anteroposterior chest radiography can identify the position of endotracheal tube. If doubt persists, direct view of the tracheal rings by fiberoptic bronchoscopy can be a 'gold standard' to identify the position. Additionally, ultrasound may verify whether the tube is in the trachea $(20,21)$, and indirectly may detect whether a unilateral bronchial intubation exists $(22,23)$.

\section{Others}

Endotracheal tube care is an important part that has not been mentioned in other guidelines, which includes but is not limited to the following: adequate tube fixation in case of dislocation; maintaining a cuff pressure between 25-30 $\mathrm{cmH} 2 \mathrm{O}$ which is high enough to help avert aspiration and air leakage, and, at the same time, low enough to avoid airway mucosal injury; scheduled or asneeded suctioning to prevent mucus plugging; finally, stabilizing hemodynamics during the treatment of postintubation sedation.

\section{Airway management cart}

Airway management in the emergency room is unstable and unpredictable, therefore, we strongly recommend a wellstocked Airway Management Cart containing different levels of airway management devices should be readily available to ensure rapid and flexible airway management. The following allocation is recommended: (I) rigid laryngoscope with all sizes of blades; (II) video laryngoscope; (III) endotracheal tubes of all sizes; (IV) endotracheal catheter guide: common stylet, visualized tube stylet, lighted stylet, etc.; (V) supraglottic airway (LMA, intubating LMA); (VI) fiberoptic device; (VII) cricothyrotomy and (possibly) tracheotomy kit(s); (VIII) $\mathrm{ETCO}_{2}$ detection devices; (IX) ET tube holders; $(\mathrm{X})$ suction catheters; (XI) oral and nasal airways.

\section{Acknowledgements}

Thanks for all members of Chinese Collaboration Group for Emergency Airway Management. All members are listed in the Supplementary Material.

Funding: National Key Clinical Speciality Construction Project (2012-650).

\section{Footnote}

Conflicts of Interest: The authors have no conflicts of interest to declare.

\section{References}

1. Yu BW, Wu XM, Zuo MZ, et al. Difficult Airway Management Guidelines. J Clini Anesth 2013;29:93-8.

2. Langeron $\mathrm{O}$, Masso E, Huraux $\mathrm{C}$, et al. Prediction of difficult mask ventilation. Anesthesiology 2000;92:1229-36.

3. Han R, Tremper KK, Kheterpal S, et al. Grading scale for mask ventilation. Anesthesiology 2004;101:267.

4. Sellick BA. Cricoid pressure to control regurgitation of stomach contents during induction of anaesthesia. Lancet 
1961;2:404-6.

5. Vanner RG, Pryle BJ. Regurgitation and oesophageal rupture with cricoid pressure: a cadaver study. Anaesthesia 1992;47:732-5.

6. Landsman I. Cricoid pressure: indications and complications. Paediatr Anaesth 2004;14:43-7.

7. Zeidan AM, Salem MR, Mazoit JX, et al. The effectiveness of cricoid pressure for occluding the esophageal entrance in anesthetized and paralyzed patients: an experimental and observational glidescope study. Anesth Analg 2014;118:580-6.

8. Georgescu A, Miller JN, Lecklitner ML. The Sellick maneuver causing complete airway obstruction. Anesth Analg 1992;74:457-9.

9. Wilson ME, Spiegelhalter D, Robertson JA, et al. Predicting difficult intubation. Br J Anaesth 1988;61:211-6.

10. Reed MJ, Dunn MJ, McKeown DW. Can an airway assessment score predict difficulty at intubation in the emergency department? Emerg Med J 2005;22:99-102.

11. Samsoon GL, Young JR. Difficult tracheal intubation: a retrospective study. Anaesthesia 1987;42:487-90.

12. Cormack RS, Lehane J. Difficult tracheal intubation in obstetrics. Anaesthesia 1984;39:1105-11.

13. Pennant JH, White PF. The laryngeal mask airway. Its uses in anesthesiology. Anesthesiology 1993;79:144-63.

14. American Society of Anesthesiologists Task Force on Management of the Difficult Airway. Practice guidelines for management of the difficult airway: an updated report by the American Society of Anesthesiologists Task Force on Management of the Difficult Airway. Anesthesiology 2003;98:1269-77.

15. Keller C, Brimacombe J, Bittersohl J, et al. Aspiration and

Cite this article as: Sun F, Wang Y, Ma S, Zhu H, Yu X, Xu $\mathrm{J}$; on behalf of Chinese Collaboration Group for Emergency Airway Management. Clinical consensus of emergency airway management. J Thorac Dis 2017;9(11):4599-4606. doi: $10.21037 /$ jtd.2017.10.79 the laryngeal mask airway: three cases and a review of the literature. Br J Anaesth 2004;93:579-82.

16. Melker JS, Gabrielli A. Melker cricothyrotomy kit: an alternative to the surgical technique. Ann Otol Rhinol Laryngol 2005;114:525-8.

17. Brunel W, Coleman DL, Schwartz DE, et al. Assessment of routine chest roentgenograms and the physical examination to confirm endotracheal tube position. Chest 1989;96:1043-5.

18. McElwain J, Malik MA, Harte BH, et al. Comparison of the C-MAC videolaryngoscope with the Macintosh, Glidescope, and Airtraq laryngoscopes in easy and difficult laryngoscopy scenarios in manikins. Anaesthesia 2010;65:483-9.

19. Birmingham PK, Cheney FW, Ward RJ. Esophageal intubation: a review of detection techniques. Anesth Analg 1986;65:886-91.

20. Chou HC, Tseng WP, Wang CH, et al. Tracheal rapid ultrasound exam (T.R.U.E.) for confirming endotracheal tube placement during emergency intubation.

Resuscitation 2011;82:1279-84.

21. Gottlieb M, Bailitz JM, Christian E, et al. Accuracy of a novel ultrasound technique for confirmation of endotracheal intubation by expert and novice emergency physicians. West J Emerg Med 2014;15:834-9.

22. Rudraraju P, Eisen LA. Confirmation of endotracheal tube position: a narrative review. J Intensive Care Med 2009;24:283-92.

23. Sim SS, Lien WC, Chou HC, et al. Ultrasonographic lung sliding sign in confirming proper endotracheal intubation during emergency intubation. Resuscitation 2012;83:307-12. 